\title{
Auditory Information Processing During Adequate Propofol Anesthesia Monitored by Electroencephalogram Bispectral Index
}

\author{
Chantal Kerssens, MA*+, Jan Klein, MD, PhD十, Andries van der Woerd, MDt, and \\ Benno Bonke, PhD* \\ *Department of Medical Psychology and Psychotherapy, Netherlands Institute of Health Sciences, Erasmus University \\ Rotterdam, the Netherlands; and +Department of Anesthesia, University Hospital Rotterdam, the Netherlands
}

\begin{abstract}
Memory for intraoperative events may arise from inadequate anesthesia when the hypnotic state is not continuously monitored. Electroencephalogram bispectral index (BIS) enables monitoring of the hypnotic state and titration of anesthesia to an adequate level (BIS 40 to 60). At this level, preserved memory function has been observed in trauma patients. We investigated memory formation in elective surgical outpatients during targetcontrolled propofol anesthesia supplemented with alfentanil. While BIS remained between 40 and 60, patients listened to a tape with either familiar instances (exemplars) from two categories (Experimental [E] group, $n=41$ ) or bird sounds (Control [C] group, $n=$
\end{abstract}

41). After recovery, memory was tested directly and indirectly. BIS during audio presentation was on average ( \pm SD) $44 \pm 5$ and $46 \pm 5$ for Groups E and C, respectively. No patient consciously recalled the intraoperative period, nor were presented words recognized reliably (Group E, $0.9 \pm 0.8$ hits; Group C, $0.8 \pm 0.8$ hits) ( $P$ $=0.7$ ). When asked to generate category exemplars, Group E named $2.10 \pm 1.0$ hits versus $1.98 \pm 1.0$ in Group C $(P=0.9)$. We found no explicit or implicit memory effect of familiar words presented during adequate propofol anesthesia at BIS levels between 40 and 60 in elective surgical patients.

(Anesth Analg 2001;92:1210-4)
$\mathbf{V}$ arious studies have demonstrated implicit (unconscious) memory effects for auditory stimuli presented during general anesthesia (1-4). Such information processing, however, may result from temporary lightening of anesthesia whenever the depth of anesthesia is not continuously monitored during stimulus presentation. Also, most of the commonly used memory tests do not differentiate between conscious and unconscious contributions to memory performance $(5,6)$. Therefore, it remains unclear to what extent auditory information is processed during general anesthesia. This is not only important with respect to the increasing number of patients claiming recall under anesthesia, but particularly so for the emotional well-being of surgical patients $(7,8)$.

Electroencephalographic (EEG) bispectral index (BIS) is an on-line measure of hypnotic state and a

Supported in part by Erasmus University Rotterdam. The A-1000 electroencephalogram monitor was borrowed from Aspect Medical Systems Inc., Newton, MA.

Accepted for publication January 23, 2001.

Address correspondence and reprint requests to Ms. Chantal Kerssens, Department of Medical Psychology and Psychotherapy, Erasmus University Rotterdam, P.O. Box 1738, NL-3000 DR, Rotterdam. Address e-mail to kerssens@mpp.fgg.eur.nl. reportedly valuable predictor of consciousness and recall with various anesthetic regimens (9-16). BIS ranges from 100 (awake) to 0 (isoelectric brain). Values between 40 and 60 indicate adequate general anesthesia, reflected by absence of response to command, alertness, and recall. Furthermore, titration of anesthesia to BIS 40-60 appears clinically useful in terms of anesthetic requirement and recovery (17-19). Recently, BIS was related to memory function in trauma patients (20). Reliable evidence for implicit memory indicated that information was partially processed, even at BIS levels between 40 and 60. In elective surgical patients, no memory was reported for an auditory story presented at these levels (19), but memory assessment was flawed because no base rate performance was established.

To investigate information processing during adequate anesthesia for elective surgical procedures, we exposed surgical outpatients to auditory stimuli while BIS remained between 40 and 60. Given our hypothesis that information processing is preserved during controlled adequate anesthesia, we expected implicit memory effects in the absence of conscious recall. We used the same test that reliably demonstrated implicit 
memory in two previous studies, in which hypnotic state had not been monitored $(21,22)$.

\section{Methods}

After study approval by the local institutional human investigation committee, written informed consent was obtained from 102 elective surgical outpatients (ASA I and II) at the University Hospital Rotterdam, the Netherlands. Patients were between 18 and $65 \mathrm{yr}$ of age, fluent in Dutch, and reported not to have hearing impairment, alcohol or drug abuse, or psychiatric illness. Eighteen patients were excluded from data analyses because stimulus processing during anesthesia was theoretically unlikely caused by deep anesthesia (BIS $<40$ during all word presentations, $n$ = 9; mean BIS $+1 \mathrm{sD}$ below 40 , resulting in approximately $85 \%$ of presentations below 40 assuming normal distribution, $n=9$ ). In this group of patients, there was not enough time for BIS to increase once anesthesia had been induced to a deep level (BIS < 40). One patient was excluded because stimulus processing was theoretically likely caused by inadvertent lightening of anesthesia (BIS $>60$ during at least $10 \%$ of word presentations), and another one caused by the use of psychoactive medication. The remaining 82 patients comprised the Experimental $(\mathrm{E})(n=41)$ and Control (C) $(n=41)$ groups.

Electrical brain activity was measured by an A1000 monitor (Aspect Medical Systems, Newton, MA) by using a two-channel referential montage and four selfpreparing electrodes (Zipprep, Aspect Medical Systems) attached above the left and right outer malar bone (At1 and At2), high on the forehead (Fpz, reference), and approximately $2 \mathrm{~cm}$ to the right of the reference electrode (Fp2, ground). Electrode impedance was $<5 \mathrm{k} \Omega$. Recordings of BIS (version 3.2) started before the induction of anesthesia and continued until anesthetic emergence.

Patients arrived in the operating room unpremedicated, where they received IV anesthesia with propofol. Target-controlled anesthesia was accomplished by using an infusion pump (Ivac; Alaris Medical Systems, San Diego, CA) incorporating a pharmacokinetic model (Diprifusor; AstraZeneca Macclesfield, Cheshire, UK) targeting propofol plasma concentrations. Target concentration was set at $6 \mu \mathrm{g} / \mathrm{mL}$ for the induction. After loss of the eyelash reflex, the lungs were ventilated with $100 \%$ oxygen. When BIS decreased $<70$, a bolus of alfentanil $(0.02 \mathrm{mg} / \mathrm{kg})$ and vecuronium bromide $(0.1 \mathrm{mg} / \mathrm{kg})$ was injected IV, after which the lungs were mechanically ventilated by laryngeal mask with a mixture of air and oxygen (40\%:60\%). Propofol plasma concentrations were titrated to BIS values between 40 and 60 to maintain adequate hypnosis during surgery in general and presentation of auditory stimuli in particular. Target concentration (in $\mu \mathrm{g} / \mathrm{mL}$ ) was adjusted in the following successive steps: 6,5 , $4.5,4,3.5,3,2.8,2.6$, and so on in steps of $0.2 \mu \mathrm{g} / \mathrm{mL}$, and vice versa.

Experimental stimuli were similar to those used in two previous studies that demonstrated an implicit memory effect for four common exemplars of familiar word categories presented during general anesthesia $(21,22)$. In these studies, patients listened to the repeated recordings of yellow, banana, green, pear (E group) or to seaside sounds (C group) and were asked after surgery to name the first three fruits and colors coming to mind. Without conscious recall, exemplars presented during anesthesia ("hits") were generated significantly more often by patients in the E group. A large group difference was observed in the first study with this test, with averages of 2.35 vs 0.79 hits (effect size, $d=1.6)(21)$. A replication study resulted in a smaller, yet significant, memory effect, with averages of 2.4 vs 1.84 hits $(d=0.6)(22)$. Given these effect sizes, the a priori probability to demonstrate a memory effect if there is a genuine difference between conditions is between 0.84 and 1.00 when 41 patients are included in either group.

Accordingly, we randomly assigned patients to Group E or C and presented stimuli on audiotape from the first incision onward. For Group C, the tape contained $45 \mathrm{~min}$ of filler sound (birds singing). For Group E, the tape started with filler sound ( $3 \mathrm{~min}$ ) followed by the repeated recordings $(15 \mathrm{~min}$ ) of the four exemplars, after which filler sounds continued. Target exemplars were recorded in a female voice (experimenter CK) at a speed of one word every $1.5 \mathrm{~s}$, and series were repeated 30 times with $20 \mathrm{~s}$ silence between repetitions. Tapes were coded by someone not involved in the experiment to ensure a doubleblinded study.

To assess memory for the intraoperative period in general and the four presented words in particular, patients were interviewed at the earliest convenient time after surgery. The interview assessed conscious recall of the surgical period by asking patients what they remembered ("what is the last thing you remember before you fell asleep?" "What is the first thing you remember after waking up?" "Do you remember anything in between?" "Did you dream?"). This is also referred to as a direct, conscious, or explicit memory test. Because memory may be implicit (unconscious), however, it is preferable to administer additional tests that do not require conscious recollection. Accordingly, we used a recognition test and category exemplar generation task. Both may reveal implicit memory effects, provided all items are responded to (4). The former is a less stringent test of implicit memory, however, because it makes reference to the learning episode (i.e., direct memory test), whereas the latter does not (i.e., indirect memory test). 
After the interview, we administered the exemplar generation task and asked patients to name the first three exemplars coming to mind for the categories vegetables, fruits, and colors (in that order). Exemplar generation from the first category assessed whether groups responded similarly to a new category of which exemplars had not been presented to either group (control assessment). In contrast, exemplar generation from the latter two categories tested memory for exemplars presented to Group E during anesthesia (experimental assessment). In the absence of conscious recall, implicit memory would be evident if Group E generated more target exemplars than Group C. Finally, word recognition was measured by reading out loud four exemplars from each category. For fruits and colors, these consisted of the two target exemplars presented to Group E during anesthesia (banana, pear, yellow, green) and two exemplars that had not been presented to either group (apple, orange, red, blue). From each category, patients were instructed to choose one exemplar they possibly recognized from the anesthetic period or to guess otherwise (fouralternative forced choice). Recognition memory would be evident if patients in Group E identified more target exemplars than patients in Group C. In the absence of conscious recall, this would signal implicit memory.

Cluster analysis explored the multiple response set for vegetable exemplar generation in Groups E and C. $\chi^{2}$ tests were used to analyze observed frequency distributions (target exemplar generation and recognition, sex, surgery type), and independent samples Student's $t$-tests were used to analyze continuous data (age; duration of surgery and anesthesia; amount of anesthesia, analgesia and muscle relaxant; average BIS during audio presentation; time between end of surgery and test). A Bonferroni correction for multiple testing was applied to tests of patient characteristics, dividing the usual level of significance $(\alpha=0.05)$ by the number of tests (10). For the remaining tests, $P<$ 0.05 was considered statistically significant. Data are presented as mean $\pm \mathrm{SD}$.

\section{Results}

Patients (38 women, 44 men) were on average $35 \pm 11 \mathrm{yr}$ of age (range, 18-61 yr) and underwent either orthopedic $(n=42)$, general $(n=29)$, or urologic $(n=8)$ surgery. The $E$ and $C$ groups were comparable on relevant patient characteristics (Table 1). No patient reported conscious recollection of the intraoperative period, nor were presented exemplars recognized more often by patients in Group E $(0.9 \pm 0.8$ hits $)$ than in Group C (0.8 \pm 0.8 hits $)(P=0.7)$. To control for a priori group differences in task performance, patients generated exemplars from a control category (vegetables). Responses were effectively described by a two-dimensional solution, indicating that two main response clusters were discriminated: one predominated by common vegetable exemplars and the other by less common exemplars. Individual patient loadings on either dimension indicated that both groups were equally inclined to generate common category exemplars. When asked to name exemplars from the experimental categories, Group E generated $2.10 \pm 1.0$ hits compared with $1.98 \pm 1.0$ hits in Group C $(P=0.9)$. Effect size (d) was 0.12 .

A post hoc multiple linear regression analysis explored potential confounding of performance on the experimental category exemplar generation task. Hit scores consistently varied with the duration of anesthesia $(r=0.25, P<0.05)$ and type of surgery $(r=$ $0.33, P<0.01)$. Correlations indicated that more hits were generated after prolonged anesthesia, as well as after urologic (2.82 \pm 0.9$)$ and orthopedic (2.10 \pm 1.0$)$ surgery than after general surgery $(1.66 \pm 1.0)$. Anesthesia and surgery had a similar effect on performance in Groups E and C. This is indicated by a largely unaffected regression weight $(\beta$, measure of validity and effect size) and SE (reflecting reliability) in the prediction of study group hit scores when covariates were $(\beta=0.04, \mathrm{SE}=0.11)$ or were not included $(\beta=$ $0.06, \mathrm{SE}=0.11)$ in the regression model.

Propofol target concentration during audiotape presentation was $3.6 \pm 0.6 \mu \mathrm{g} / \mathrm{mL}$ in Group E and $3.7 \pm$ $0.8 \mu \mathrm{g} / \mathrm{mL}$ in Group C $(P=0.3)$, which resulted in clustering of hypnotic states near BIS 40 (Fig. 1). As can be seen in Figure 2, an overall stable level of hypnosis was obtained during word presentation to patients in Group E. Post hoc exploration of the relation between mean BIS during word presentation and target exemplar generation in Group E revealed no decrease in hit scores with increasing hypnotic depth at this controlled level of hypnosis (BIS 55-50, 1.8 hits, $n=5$; BIS 50-45, 1.9 hits, $n=9$; BIS 45-40, 1.9 hits, $n$ $=13$; BIS $40-35,2.6$ hits, $n=14$ ). No statistical analyses were performed because observations were unevenly distributed and limited in number.

\section{Discussion}

Implicit memory effects for auditory information presented during general anesthesia imply information processing during anesthesia. Because such effects may result from inadequate anesthesia, however, it is important to control the level of hypnosis during stimulus presentation. This study used EEG-BIS to monitor the hypnotic state continuously while a series of words was repeatedly presented to patients in the $\mathrm{E}$ group. More important, words were presented during adequate anesthesia only, reflected by BIS values between 40 and 60. Patients in the $C$ group were anesthetized similarly but heard filler sounds instead. Implicit memory effects for presented words had been demonstrated in two previous, but similar, studies without controlling for hypnotic state $(21,22)$. Therefore, we used the same study material but presented stimuli under 
Table 1. Patient Characteristics

\begin{tabular}{|c|c|c|}
\hline Characteristic & $\begin{array}{l}\text { Experimental group } \\
\qquad(n=41)\end{array}$ & $\begin{array}{c}\text { Control group } \\
\quad(n=41)\end{array}$ \\
\hline Age (yr) & $34 \pm 10$ & $36 \pm 11$ \\
\hline Men/women $(n)$ & $25 / 16$ & $19 / 22$ \\
\hline Orthopedic/general/urologic surgery $(n)$ & $19 / 14 / 8$ & $23 / 15 / 3$ \\
\hline Duration of surgery $(\mathrm{min})$ & $38 \pm 28$ & $34 \pm 23$ \\
\hline Duration of anesthesia (min) & $59 \pm 30$ & $54 \pm 23$ \\
\hline Total dose of propofol (mg) & $750 \pm 340$ & $710 \pm 280$ \\
\hline Total dose of alfentanil (mg) & $1.2 \pm 0.3$ & $1.1 \pm 0.3$ \\
\hline Total dose of vecuronium (mg) & $7.0 \pm 2.1$ & $6.6 \pm 1.3$ \\
\hline BIS during audio presentation & $44 \pm 5$ & $46 \pm 5$ \\
\hline Time until test (min) & $115 \pm 32$ & $110 \pm 39$ \\
\hline
\end{tabular}

Values are mean \pm SD or observed frequency.

Means were compared with Student's $t$-test for two independent samples (Experimental versus Control group).

Frequency distributions in groups were compared with $\chi^{2}$ tests. All differences were statistically nonsignificant.

BIS $=$ bispectral index.

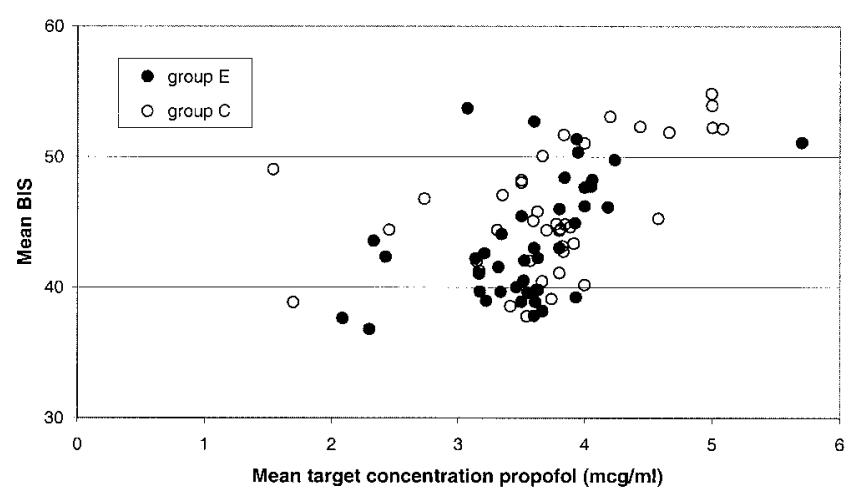

Figure 1. Bispectral index (BIS) in relation to targeted plasma concentration propofol during audio presentation in the Experimental (E) $(n=41)$ and Control (C) $(n=41)$ groups.

strict anesthetic monitoring to assess memory formation during controlled adequate anesthesia.

Although the a priori probability to detect group differences was high with this particular paradigm, no memory effect was observed. Patients in the E group displayed no conscious recall and were unable to recognize presented category exemplars. In addition, they failed to generate more hits than the $C$ group. Hence, no evidence of word priming during anesthesia was found. Given the small effect size in this sample, the probability of demonstrating an experimental effect was low in retrospect (power $=0.12$ ). More specifically, our results indicate that words presented at BIS levels near 45 were not processed to the extent that memory could occur. This is in line with reports by Struys et al. (19), who found no evidence of memory for auditory information presented at BIS levels between 40 and 60 .

Because previous studies that used the same stimuli $(21,22)$ demonstrated implicit memory effects without monitoring the hypnotic state, our null findings suggest diminished information processing when controlled adequate anesthesia is provided. At this controlled level, stimuli may be processed less elaborately

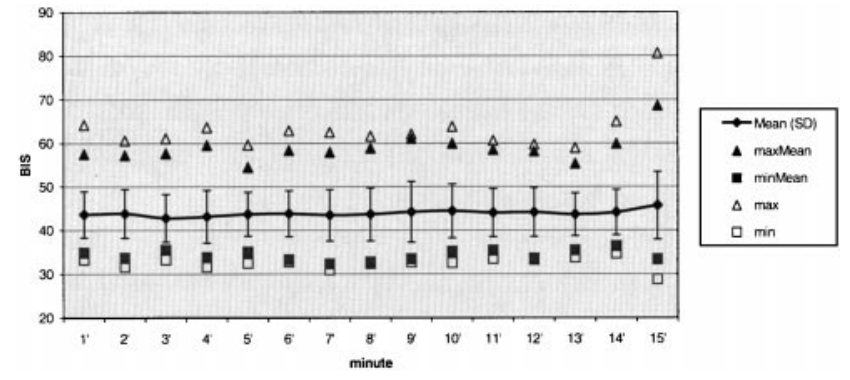

Figure 2. Bispectral index (BIS) during word presentation in the Experimental group $(n=41)$. For each minute, an average (Mean) over all BIS samples $(n=495)$ was calculated. The range of observations is indicated by the highest (max) and lowest (min) BIS value. In addition, an average BIS was calculated for each patient, the range of which is indicated by the highest (maxMean) and lowest (minMean) mean observed. Mean BIS values $>60$ were observed in the ninth minute $\left(n=2 ; \mathrm{M}_{1}=60.5, \mathrm{M}_{2}=61.1\right)$ and in the 15th min $\left(n=3 ; \mathrm{M}_{1}=63.5, \mathrm{M}_{2}=63.6, \mathrm{M}_{3}=68.6\right)$.

or by fewer individuals, resulting in reduced stimulus processing in general and absence of memory formation. This notion is again supported by Struys et al. (19), who found a decreased (zero) incidence of implicit memory for BIS-guided anesthetics compared with procedures monitoring classic signs of anesthetic adequacy. Our findings implicate that previous demonstrations of implicit memory $(21,22)$ may have come about by undetected moments of inadequate anesthesia and stress the importance of maintaining stable levels of hypnosis during surgery and stimulus presentation in particular. Together, these observations support the feasibility of BIS as a monitor of adequate anesthesia without memory formation.

This notion is not consistent with the implicit memory effect found for words presented at BIS 40 to 60 in trauma patients (20). Because that study included a wide range of hypnotic states (i.e., BIS 21 to 96), however, moments of lightened anesthesia affected results. In a similar vein, it should be noted that analyses were confined to words, i.e., relating the BIS level during word 
presentation to subsequent memory performance, thereby neglecting intraindividual variations in hypnotic state. Therefore, although a significant memory effect for words presented at adequate levels of hypnosis was found, effects of light anesthesia within the individual patient were not controlled for in the trauma study. In this study and that of Struys et al. (19), they were.

Second, differences in the anesthetic regimen may partly explain controversial memory findings at equisedative levels of hypnosis. In the trauma study (20), isoflurane was used for anesthetic maintenance, whereas both Struys et al. (19) and we used propofol, a reportedly effective anesthetic in abolition of information processing and memory $(23,24)$. A large multicenter study found no relation between anesthetic regimen and probability of recall, but curves for propofol and isoflurane appeared to have different slopes, suggesting different relations to memory (12). In particular, memory tended to occur earlier with isoflurane than with propofol. Because propofol may have contributed to the null findings, replication studies with other anesthetics are important.

A third explanation for the memory effect observed in trauma patients but not in elective surgical patients may be found in the type of surgery and associated level of stress. Increased levels of stress hormones, e.g., epinephrine and cortisol, modulate memory storage (25). Trauma patients presumably are exposed to more stress than elective surgical outpatients, which may explain why memory has been observed in the former but not in the latter group of patients at adequate levels of hypnosis.

To summarize, the lack of evidence for explicit and implicit memory in this study suggests absence of memory formation when controlled adequate propofol anesthesia is provided. Maintaining stable levels of hypnosis seems crucial in this respect. Additional studies need to establish the reliability of our observations, especially so with anesthetics other than propofol.

We thank all staff and patients from the ambulatory surgical unit (University Hospital Rotterdam) for their cooperation with the study. We also thank Aspect Medical Systems, Inc., for providing us with EEG equipment; Gitta Lubke and Peter Sebel for their comments on earlier drafts of this article; and Hugo Duivenvoorden for his statistical input.

\section{References}

1. Ghoneim MM, Block RI. Learning and consciousness during general anesthesia. Anesthesiology 1992;76:279-305.
2. Andrade J. Learning during anaesthesia: a review. Br J Psychol 1995;86:479-506

3. Ghoneim MM, Block RI. Learning and memory during general anesthesia: an update. Anesthesiology 1997;87:387-410.

4. Merikle PM, Daneman M. Memory for unconsciously perceived events: evidence from anesthetized patients. Conscious Cogn 1996;5:525-41.

5. Jacoby LL. A process dissociation framework: separating automatic from intentional uses of memory. J Mem Lang 1991;30:513-41.

6. Jacoby LL, Toth JP, Yonelinas AP. Separating conscious and unconscious influences of memory: measuring recollection. J Exp Psychol Gen 1993;122:139-54.

7. Osterman JE, van der Kolk BA. Awareness during anesthesia and posttraumatic stress disorder. Gen Hosp Psychiatry 1998;50:274-8.

8. Domino KB, Posner KL, Caplan RA, Cheney FW. Awareness during anesthesia: a closed claims analysis. Anesthesiology 1999;90:1053-61.

9. Sigl JC, Chamoun NG. An introduction to bispectral analysis for the electroencephalogram. J Clin Monit 1994;10:392-404.

10. Liu J, Singh H, White PF. Electroencephalographic bispectral analysis predicts the depth of midazolam induced sedation. Anesthesiology 1996;84:64-9.

11. Liu J, Singh H, White PF. Electroencephalographic bispectral index correlates with intraoperative recall and depth of propofol-induced sedation. Anesth Analg 1997;84:185-9.

12. Glass PS, Bloom M, Kearse L, et al. Bispectral analysis measures sedation and memory effects of propofol, midazolam, isoflurane and alfentanil in healthy volunteers. Anesthesiology 1997;86:836-47.

13. Iselin-Chaves IA, Flaishon R, Sebel PS, et al. The effect of the interaction of propofol and alfentanil on recall, loss of consciousness, and the bispectral index. Anesth Analg 1998;87:949-55.

14. Katoh T, Suzuki A, Ikeda K. Electroencephalographic derivatives as a tool for predicting the depth of sedation and anesthesia induced by sevoflurane. Anesthesiology 1998;88:642-50.

15. Kearse LA, Rosow C, Zaslavsky A, et al. Bispectral analysis of the electroencephalogram predicts conscious processing of information during propofol sedation and hypnosis. Anesthesiology 1998;88:25-34.

16. Rampil I. A primer for EEG signal processing in anesthesia. Anesthesiology 1998;89:980-1002.

17. Gan TJ, Glass PS, Windsor A, et al. Bispectral index monitoring allows faster emergence and improved recovery from propofol alfentanil and nitrous oxide anesthesia. Anesthesiology 1997;87:808-15.

18. Song D, Joshi GP, White PF. Titration of volatile anesthetics using bispectral index facilitates recovery after ambulatory anesthesia. Anesthesiology 1997;87:842-8.

19. Struys M, Versichelen L, Byttebier G, et al. Clinical usefulness of the bispectral index for titrating propofol target effect site concentration. Anaesthesia 1998;53:4-12.

20. Lubke GH, Kerssens C, Phaf H, Sebel PS. Dependence of explicit and implicit memory on hypnotic state in trauma patients. Anesthesiology 1999;90:670-80.

21. Roorda-Hrdlicková V, Wolters G, Bonke B, Phaf RH. Unconscious perception during general anaesthesia, demonstrated by an implicit memory task. In: Bonke B, Fitch W, Millar K, eds. Memory and awareness in anaesthesia. Amsterdam: Swets \& Zeitlinger, 1990:151-5.

22. Jelicic M, Bonke B, Wolters G, Phaf RH. Implicit memory for words presented during anaesthesia. Eur J Cogn Psychol 1992;4:71-80.

23. Veselis RA, Reinsel RA, Wronski M, et al. EEG and memory effects of low-dose infusions of propofol. Br J Anaesth 1992;69:246-54.

24. Veselis RA, Reinsel RA, Feshchenko VA, Wronski M. The comparative amnesic effects of midazolam, propofol, thiopental and fentanyl at equisedative concentrations. Anesthesiology 1997;87:749-64.

25. Cahill L, McGaugh JL. Mechanisms of emotional arousal and lasting declarative memory. Trends Neurosci 1998;21:294-9. 